\title{
Burnout Syndrome and Consumption of Alcohol and Illicit Substances in University Students
}

\author{
Giovana Frazon Andrade ${ }^{1}$ \\ Poliana Vieira da Silva Menolli ${ }^{2}$ (D) \\ Pedro Augusto Clemente ${ }^{1}$ (I) \\ Arthur Eumann Mesas ${ }^{3}$ \\ Dannyele Cristina Silva ${ }^{1}$ \\ Edmarlon Girotto ${ }^{4}$
}

\begin{abstract}
The consumption of alcohol and illicit substances has shown alarming levels among young university students, and its use may be associated with the coping strategy of academic burnout. This review aimed to synthesize the scientific evidence related to the consumption of alcohol and illicit substances associated with burnout syndrome in university students. Searches were carried out in: Biblioteca Virtual em Saúde, PubMed, Web of Science, PsycNet and Scopus databases, with no year limitation and based on the PRISMA guideline. Among the 2.269 articles identified, 16 met the inclusion criteria. All studies evaluated students in the health area, 12 studies found an association between the variables, with alcohol and illicit substances consumption strongly related to burnout syndrome. It was concluded that there is an association between the studied variables, indicating that the presence of burnout syndrome in university students can result in the use of negative strategies as a coping measure.
\end{abstract}

Keywords: drug usage, alcohol abuse, students, coping behavior, occupational stress

\section{Síndrome de Burnout e Consumo de Álcool e Substâncias Ilícitas em Universitários}

\begin{abstract}
Resumo: O consumo de álcool e substâncias ilícita tem apresentado níveis alarmantes entre jovens universitários, e seu uso pode estar associado à estratégia de enfrentamento do burnout acadêmico. Esta revisão teve por objetivo sintetizar as evidências científicas relacionadas ao consumo de álcool e substâncias ilícitas associadas a síndrome de burnout em estudantes universitários. Foram realizadas buscas nas bases: Biblioteca Virtual em Saúde, PubMed, Web of Science, PsycNet e Scopus, sem limitação de ano e baseado na guia PRISMA. Entre os 2.269 artigos identificados, 16 atenderam aos critérios de inclusão. Todos os estudos avaliaram estudantes da área da saúde, 12 estudos encontraram associação entre as variáveis, sendo o consumo de álcool e substâncias ilícitas fortemente relacionado à sindrome de burnout. Concluiu-se que há associação entre as variáveis estudadas, indicando que a presença da síndrome de burnout em universitários pode resultar no uso de estratégias negativas como medida de enfrentamento.
\end{abstract}

Palavras-chave: droga (uso), abuso de álcool, estudantes, enfrentamento, stress ocupacional

\section{Síndrome de Burnout y Consumo de Alcohol y de Sustancias Ilícitas por Universitarios}

\begin{abstract}
Resumen: El consumo de alcohol y de sustancias ilícitas ha mostrado niveles alarmantes entre los jóvenes universitarios, y su uso puede estar asociado con estrategias de enfrentamiento del burnout académico. Esta revisión tuvo como objetivo sintetizar la evidencia científica relacionada con el consumo de alcohol y de sustancias ilícitas asociadas al síndrome de burnout en estudiantes universitarios. Las búsquedas se realizaron en las bases Biblioteca Virtual en Salud, PubMed, Web of Science, PsycNet y Scopus, sin limitación de año y basándose en la guía PRISMA. De los 2.269 artículos encontrados, 16 cumplieron los criterios de inclusión. Todos los estudios evaluaron estudiantes del área de salud y 12 estudios encontraron asociación entre las variables, entre los que el consumo de alcohol y de sustancias ilícitas estuvo fuertemente relacionado con el síndrome de burnout. Se concluyó que existe asociación entre las variables estudiadas, indicando que la presencia de síndrome de burnout en estudiantes universitarios puede resultar en el uso de estrategias negativas como medida de enfrentamiento.
\end{abstract}

Palabras clave: drogas (uso), abuso de alcohol, estudiantes, enfrentamiento, estres ocupacional

\footnotetext{
${ }^{I}$ Universidade Estadual do Centro-Oeste, Guarapuava-PR, Brazil

${ }^{2}$ Universidade Estadual do Oeste do Paraná, Cascavel-PR, Brazil

${ }^{3}$ Universidad de Castilla-La Mancha, Cuenca, Espanha

${ }^{4}$ Universidade Estadual de Londrina, Londrina-PR, Brazil
}

Article derived from the doctoral thesis by Giovana Frazon Andrade under the supervision of Dr. Edmarlon Girotto, with defense scheduled for 2021, in the Graduate Program in Public Health at the Universidade Estadual de Londrina. Correspondence address: Giovana Frazon Andrade. Universidade Estadual do Centro-Oeste. Alameda Élio Antonio Dalla Vechia, 838, Guarapuava-PR, Brazil. CEP 85.015-430. E-mail: giovanafrazon@hotmail.com
The consumption of alcohol and illicit substances in the world has shown alarming levels, and this abuse is a major public health issue. According to the World Health Organization, it is estimated that, currently, $43 \%$ of the world population aged 15 years and over consume alcoholic beverages (World Health Organization [WHO], 2018). In relation to illicit substances, the "World Report on Drugs 2020," produced by the United Nations, estimated that 269 million people in the world have already consumed some type 
of drug, characterizing an increase of 30\% compared to 2009 (United Nations Office on Drugs and Crime [UNODC], 2020).

The consumption of alcohol and illicit substances in various social groups is evident (Benincasa et al., 2018; Munhoz et al., 2017; Trevisan \& Castro, 2019) and a category that deserves notoriety are university students, due to the worrying prevalence of consumption (Aresi, Moore, \& Marta, 2016; Davoren, Demant, Shiely, \& Perry, 2016; Newman, Ding, \& Feng, 2017). To understand the problem in this population group, it is essential to identify the risk factors for its consumption. One of the interpretations comes from the fact that students are in a vulnerable situation in the development of intellectual and emotional conflicts due to confrontations in the academic environment (Ariño \& Bardagi, 2018). Among these, high competitiveness among students, excess of curricular and extracurricular activities, lack of time for leisure activities with friends and family stand out, in addition to personal needs and concerns about the professional future (Ariño \& Bardagi, 2018; Pacheco et al. al., 2017).

From this perspective, burnout syndrome (BS) arises as a result of the worsening of these stressful effects in which the student is inserted. It is defined as a psychological syndrome of emotional exhaustion characterized in three dimensions: Emotional Exhaustion (EE) - lack of willingness for activities and emotional exhaustion due to the demands of studies; Depersonalization (DP) - lack of sensitivity and tendency to react in a cold and detached way; and low Professional Achievement (PA) - feeling of dissatisfaction and incompetence in relation to studies (Maslach, Jackson, \& Leiter, 1997). The university student seeks ways of coping with the feeling of exhaustion, and the use of psychoactive substances is a negative strategy for coping with these situations (M.S. Carlotto, Câmara, Otto, \& Kauffmann, 2010; Erschens et al., 2018; Njim et al., 2019).

Given this context, it is noticeable that, among the reasons that lead college students to consume alcohol and illicit substances, directing the view from the perspective of academic burnout becomes necessary as further deepening of this problem can help in actions aimed at students' mental health. Furthermore, what exists in the literature is dispersed, and the synthesis of this evidence will have implications for the establishment of priorities in future studies on the subject. This review aimed to synthesize the scientific evidence related to the consumption of alcohol and illicit substances associated with burnout syndrome in university students.

\section{Method}

This is a systematic review of studies on the relationship between BS and the consumption of alcoholic beverages and/or illicit substances by university students. The inclusion criteria were: original studies with specific quantitative results on the relationship that is object of the review, and published in Portuguese, English or Spanish. No publication date limits were applied. The recommendations of the Preferred Reporting Items of Systematic Reviews and
Meta-Analyses (PRISMA) were followed. Searches were performed in the selected databases Virtual Health Library (VHL), PubMed, Web of Science, PsycNet and Scopus, and articles published until January 1, 2021 were included. The key search terms used were: alcohol*, drink*, crack, marijuana, cannabis, cocaine, amphetamine*, psychoactive*, stimulant*, substance*, drug*, hallucinog*, heroin*, ecstasy, inhalant*, burnout, accomplishment, depersonalization, exhaustion, undergraduate*, student*, universit*, graduate* e academic*. We considered the key terms present in the title, abstract and subject for the VHL database, title and abstract for the PubMed database, title and topic for the Web of Science database, abstract for PsycNet, and title, abstract and descriptors for Scopus. The investigation relied on appropriate language and syntax for each database.

The entire article selection and review process were carried out independently by two researchers, who compared their results in each of the stages, resolving discrepancies by consensus. A third researcher was consulted to deliberate in case of disagreement. Duplicates were initially excluded, then the relevance of the topic was assessed by reading the titles and abstracts, discarding studies whose titles and/or abstracts clearly indicated that they were not related to the topic. After this step, the articles were read in full, checking if they met the inclusion criteria. Furthermore, the references of the selected studies were examined in order to identify publications that were not located in the previous steps and that met the inclusion criteria.

The articles included followed to the data collection and filing process. In the records, the data extracted from each study included: first author, year of publication, year of research, geographic location (country), population data (sample size), undergraduate course analyzed, collection data (variables evaluated and collection instruments used), and main results of the associations between variables. The quality (risk of bias) of each study that met the inclusion criteria was assessed using the assessment checklist of the Quality Assessment Tool for Observational Cohort and Cross-Sectional Studies published by the US National Heart, Lung and Blood Institute (https://www.nhlbi.nih.gov/healthtopics/study-quality-assessment-tools). This tool presents a checklist of fourteen items, which were applied to all studies, and performed by two independent reviewers and, in case of disagreements, a third evaluator was consulted. The final quality rating for each analysis was performed qualitatively and categorized as "poor," "fair" or "good."

\section{Results}

Figure 1 shows the research strategy and selection of the included studies. The initial search identified 2,268 articles, 1,037 in the VHL, 171 in PubMed, 354 in Web of Science, 128 in PsycNet and 578 in Scopus. A study was added by reviewing the reference lists of selected articles, resulting in a total of 2,269 articles. After eliminating duplicates and tracking by title and abstract, 67 articles were selected for full 
reading review, 51 articles were excluded with justification (six literature reviews, four with descriptive data only, one in French, thirty-seven that did not perform analysis between the studied variables and three with restricted access). In the end, 16 studies were included in this review. Regarding the study design, all researches are cross-sectional.

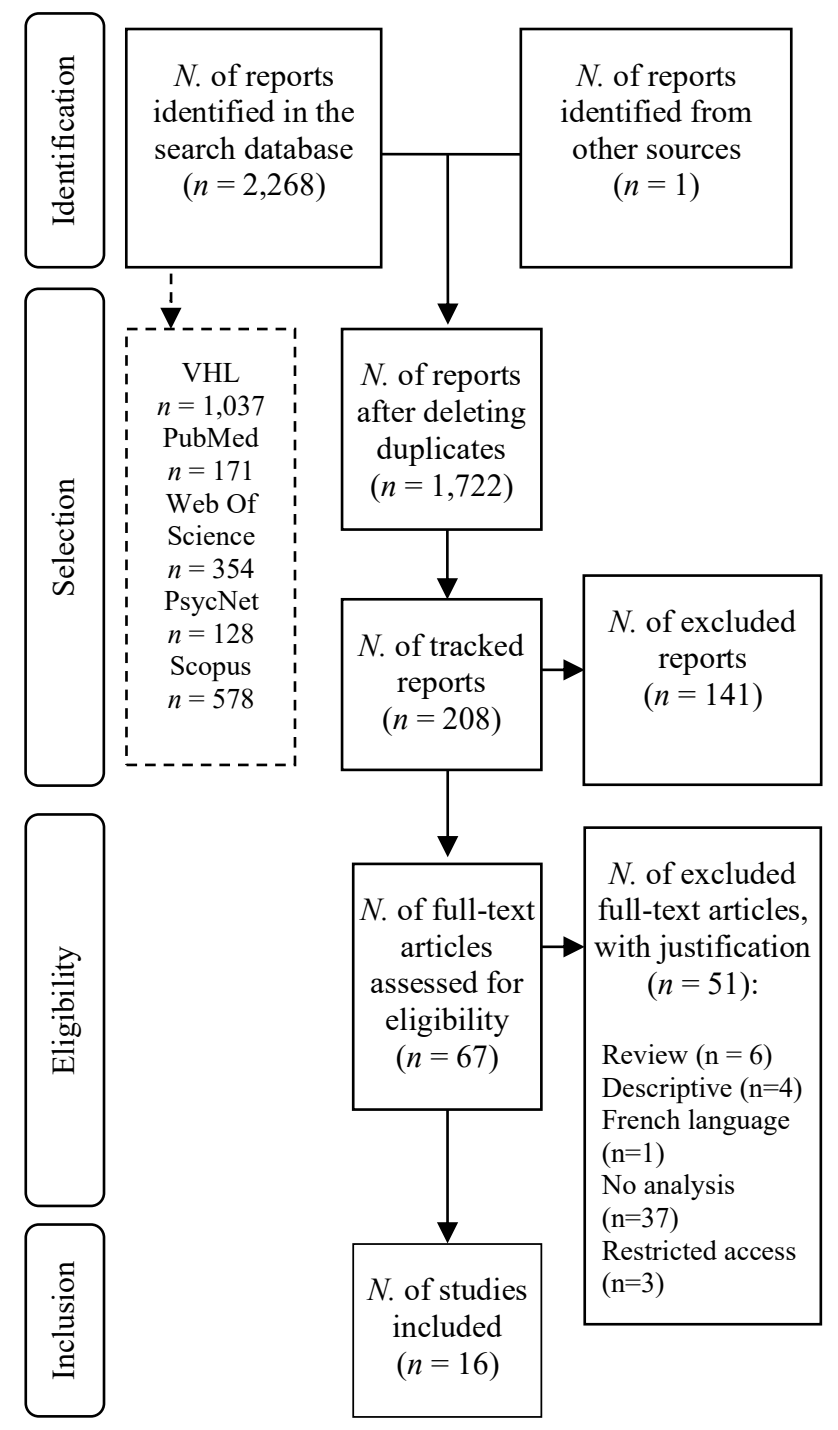

Figure 1. Flowchart of the research strategy and selection of included studies.

As for the quality analysis of the articles, nine studies were classified as having "good" quality (Arango Agudelo et al., 2010; Calcides, Didou, Melo, \& Oliva-Costa, 2019; Erschens et al., 2018; Lee et al., 2020; Njim et al., 2019, 2018; Nteveros et al., 2020; Talih, Daher, Daou, \& Ajaltouni, 2018;
Tavares et al., 2020) and seven studies rated as "fair" (M.S. Carlotto et al. al., 2010; Cecil, McHale, Hart, \& Laidlaw, 2014; Jackson, Shanafelt, Hasan, Satele, \& Dyrbye, 2016; Mache, Vitzthum, \& Groneberg, 2015; Mbanga, Efie, Aroke, \& Njim, 2018; Vilchez-Cornejo et al., 2016; Zivojinović et al., 2020). All studies had clear objectives, uniform recruitment and population eligibility criteria, validated and/ or accurate exposure and assessment measures, and results defined in detail. However, some studies showed weaknesses in the participation of the population (M.S. Carlotto et al., 2010; Cecil et al., 2014; Jackson et al., 2016) and justification for the sample size (M.S. Carlotto et al., 2010; Jackson et al., 2016; Lee et al., 2020; Mache et al., 2015; Mbanga et al., 2018; Nteveros et al., 2020; Tavares et al., 2020; Vilchez-Cornejo et al., 2016), in the presentation of different levels of exposure (Calcides et al., 2019; MS Carlotto et al., 2010; Cecil et al., 2014; Mbanga et al., 2018; Njim et al., 2018, 2019; Vilchez-Cornejo et al., et al., 2019; al., 2016; Zivojinović et al., 2020), and the application of adjustment variables in the statistical analyses (Arango Agudelo et al., 2010; M.S. Carlotto et al., 2010; Erschens et al., 2018; Mache et al., 2010; ., 2015; Nteveros et al., 2020; Vilchez-Cornejo et al., 2016).

Table 1 shows the characteristics of the studies that verified the consumption of alcohol and illicit substances associated with burnout syndrome. The articles were published between 2010 and 2020, and conducted in countries located on the following continents: five in South America (Arango Agudelo et al., 2010; Calcides et al., 2019; M.S. Carlotto et al., 2010; Tavares et al., 2020; Vilchez-Cornejo et al., 2016), with three in Brazil (Calcides et al., 2019; M.S. Carlotto et al., 2010; Tavares et al., 2020), five in Europe (Cecil et al., 2014; Erschens et al., 2018; Mache et al., 2015; Nteveros et al., 2020; Zivojinović et al., 2020), two in Asia (Lee et al., 2020; Talih et al., 2018), three in Africa (Mbanga et al., 2018; Njim et al., 2018, 2019) and one in North America (Jackson et al., 2016). As for population, most studies were conducted with samples of undergraduate students below 900 individuals (ranging from 176 to 852), and only one study was conducted with a high number of students, as it collected data from several universities $(n=4,402)$ (Jackson et al., 2016). All studies were carried out in undergraduate courses in the health area, and the Medicine course was the most prevalent $(N=11)$ (Arango Agudelo et al., 2010; Calcides et al., 2019; Cecil et al., 2014; Erschens et al., 2018; Jackson et al., 2016; Lee et al., 2020; Njim et al., 2019; Nteveros et al., 2020; Talih et al., 2018; Tavares et al., 2020; Vilchez-Cornejo et al., 2016), followed by Medicine and Nursing $(N=1)$ (Mbanga et al., 2018), Nursing $(N=1)$ (Njim et al., 2018), Psychology $(N=1)$ (M.S. Carlotto et al., 2010), Dentistry $(N=1)$ (Mache et al., 2015) and Veterinary Medicine $(N=1)$ (Zivojinović et al., 2020). 
Table 1

Characteristics of studies that verified the use of alcohol and illicit substances associated with burnout syndrome

\begin{tabular}{|c|c|c|c|c|c|}
\hline Author (year) & Date of study & Location & Population & Undergraduate course & Variables and collection instrument \\
\hline $\begin{array}{l}\text { Arango Agudelo } \\
\text { et al. (2010) }\end{array}$ & 2009 & Colombia & $\begin{aligned} N & =234 \\
\text { Male } & =53.2 \% \\
\text { Fem } & =46.8 \%\end{aligned}$ & Medicine & $\begin{array}{l}\text { BS (MBI-SS), alcohol consumption (CAGE), } \\
\text { psychoactive substances (Y/N, which one) }\end{array}$ \\
\hline $\begin{array}{l}\text { Calcides et al. } \\
\text { (2019) }\end{array}$ & 2018 & Brazil & $\begin{aligned} N & =186 \\
\text { Male } & =39.1 \% \\
\text { Fem } & =60.9 \%\end{aligned}$ & Medicine & BS (MBI-SS), psychoactive substances $(\mathrm{Y} / \mathrm{N})$ \\
\hline $\begin{array}{l}\text { M.S. Carlotto } \\
\text { et al. }(2010)\end{array}$ & 2007 & Brazil & $\begin{aligned} N & =239 \\
\text { Male } & =12.6 \% \\
\text { Fem } & =87.4 \%\end{aligned}$ & Psychology & $\begin{array}{l}\text { BS (MBI-SS), alcohol and drug consumption } \\
\text { (COPE Inventory) }\end{array}$ \\
\hline $\begin{array}{l}\text { Cecil et al. } \\
\text { (2014) }\end{array}$ & 2012 & England & $\begin{aligned} N & =356 \\
\text { Male } & =34.8 \% \\
\text { Fem } & =65.2 \%\end{aligned}$ & Medicine & BS (MBI), alcohol consumption (AUQ) \\
\hline $\begin{array}{l}\text { Erschens et al. } \\
\qquad(2018)\end{array}$ & - & Germany & $\begin{aligned} N & =597 \\
\text { Male } & =37.7 \% \\
\text { Fem } & =62.3 \%\end{aligned}$ & Medicine & $\begin{array}{l}\text { BS (MBI-SS), consumption of alcohol and } \\
\text { stimulant drugs }(\mathrm{Y} / \mathrm{N})\end{array}$ \\
\hline $\begin{array}{l}\text { Jackson et al. } \\
\quad \text { (2016) }\end{array}$ & 2012 & United States & $\begin{array}{c}N=4,402 \\
\text { Male }=45.3 \% \\
\text { Fem }=54.7 \%\end{array}$ & Medicine & BS (MBI), alcohol consumption (AUDIT-C) \\
\hline $\begin{array}{l}\text { Lee et al. } \\
(2020)\end{array}$ & 2017 & China & $\begin{aligned} N & =731 \\
\text { Male } & =44.2 \% \\
\text { Fem } & =55.8 \%\end{aligned}$ & Medicine & BS (MBI), alcohol consumption (AUDIT-C) \\
\hline $\begin{array}{l}\text { Mache et al. } \\
\quad(2015)\end{array}$ & $\begin{array}{l}2012 / \\
2013\end{array}$ & Germany & $\begin{array}{c}N=239 \\
\text { Male }=36 \% \\
\text { Fem }=64 \%\end{array}$ & Dentistry & $\begin{array}{l}\text { BS (MBI), consumption of alcohol and illegal } \\
\text { substances - cannabis, cocaine, ecstasy }(\mathrm{Y} / \mathrm{N})\end{array}$ \\
\hline $\begin{array}{l}\text { Mbanga et al. } \\
\text { (2018) }\end{array}$ & 2018 & Cameroon & $\begin{aligned} N & =852 \\
\text { Male } & =31.4 \% \\
\text { Fem } & =68.6 \%\end{aligned}$ & Medicine and Nursing & $\begin{array}{l}\text { BS }(\text { OLBI }) \text {, recreational drugs - } \\
\text { cannabis }(\mathrm{Y} / \mathrm{N})\end{array}$ \\
\hline $\begin{array}{l}\text { Njim et al. } \\
\text { (2019) }\end{array}$ & 2018 & Cameroon & $\begin{aligned} N & =413 \\
\text { Male } & =45.3 \% \\
\text { Fem } & =54.7 \%\end{aligned}$ & Medicine & $\begin{array}{c}\text { BS (OLBI), alcohol and recreational drug use } \\
\text { - cannabis }(\mathrm{Y} / \mathrm{N})\end{array}$ \\
\hline $\begin{array}{l}\text { Njim et al. } \\
\quad(2018)\end{array}$ & 2018 & Cameroon & $\begin{aligned} N & =447 \\
\text { Male } & =18.8 \% \\
\text { Fem } & =81.2 \%\end{aligned}$ & Nursing & $\begin{array}{l}\text { BS (OLBI), alcohol and recreational drug use } \\
- \text { cannabis }(\mathrm{Y} / \mathrm{N})\end{array}$ \\
\hline $\begin{array}{l}\text { Nteveros et al. } \\
\quad(2020)\end{array}$ & 2020 & Cyprus & $\begin{aligned} N & =182 \\
\text { Male } & =33.5 \% \\
\text { Fem } & =66.5 \%\end{aligned}$ & Medicine & BS (MBI-SS), alcohol consumption (Y/N) \\
\hline $\begin{array}{l}\text { Talih et al. } \\
\text { (2018) }\end{array}$ & 2016 & Lebanon & $\begin{aligned} N & =172 \\
\text { Male } & =51.1 \% \\
\text { Fem } & =48.9 \%\end{aligned}$ & Medicine & $\begin{array}{l}\text { BS (BMS), alcohol consumption (AUDIT), } \\
\text { substance use (DAST-10) }\end{array}$ \\
\hline $\begin{array}{l}\text { Tavares et al. } \\
\quad(2020)\end{array}$ & - & Brazil & $\begin{aligned} N & =419 \\
\text { Male } & =35.3 \% \\
\text { Fem } & =64.7 \%\end{aligned}$ & Medicine & $\begin{array}{l}\text { BS (MBI-SS), consumption of alcohol and } \\
\text { stimulant drugs }(\mathrm{Y} / \mathrm{N})\end{array}$ \\
\hline $\begin{array}{l}\text { Vilchez-Cornejo } \\
\text { et al. (2016) }\end{array}$ & $\begin{array}{l}2014 / \\
2015\end{array}$ & Peru & $\begin{array}{c}N=583 \\
\text { Male }=50.6 \% \\
\text { Fem }=49.4 \%\end{array}$ & Medicine & BS (MBI-SS), alcohol consumption $(\mathrm{Y} / \mathrm{N})$ \\
\hline $\begin{array}{l}\text { Zivojinovic et al. } \\
\qquad(2020)\end{array}$ & 2014 & Serbia & $\begin{aligned} N & =416 \\
\text { Male } & =66.3 \% \\
\text { Fem } & =33.7 \%\end{aligned}$ & Veterinary Medicine & BS (MBI-SS), alcohol consumption ( $\mathrm{Y} / \mathrm{N})$ \\
\hline
\end{tabular}

Note . Male $=$ Male Fem $=$ female AUDIT $=$ Alcohol Use Disorders Identification Test; AUDIT-C $=$ Alcohol Use Disorders Identification Test - brief form; AUQ = Alcohol Urge Questionnaire; BMS = Burnout Measure 10-item version; DAST-10 = Drug Abuse Screen Test; MBI = Maslach Burnout Inventory; MBI-SS = Maslach Burnout Inventory - Student Survey; OLBI = Oldenburg Burnout Inventory; $\mathrm{BS}=$ Burnout syndrome; $\mathrm{Y} / \mathrm{N}=$ Yes or No.

Regarding the consumption of alcoholic beverages, different forms of assessment were used. One study used the AlcoholUseDisorders Identification Test(AUDIT)questionnaire (Talih et al., 2018) and two studies used its abbreviated form (AUDIT-C) (Jackson et al., 2016; Lee et al., 2020). The Alcohol Urge Questionnaire (AUQ) (Cecil et al., 2014), the CAGE questionnaire (Arango Agudelo et al., 2010) and the COPE inventory (M.S. Carlotto etal., 2010) were used in one study each. Most studies used the simple questioning methodology, asking whether the student consumed alcohol or not, with "yes" or "no" answers (Erschens et al., 2018; Mache et al., 2015; Njim et al., 2018, 2019; Nteveros et al., 2020; Tavares et al., 2020; VilchezCornejo et al., 2016; Zivojinović et al., 2020). The same strategy was used to assess the consumption of illicit substances (Arango Agudelo et al., 2010; Calcides et al., 2019; Erschens et al., 2018;
Mache et al., 2015; Mbanga et al., 2018; Njim et al., 2018, 2019; Tavares et al., 2020), and three studies considered the use of cannabis and tramadol as recreational drug variable, without separating the components (Mbanga et al., 2018; Njim et al., $2018,2019)$ and one study did not identify the type of stimulant substances analyzed (Tavares et al., 2020). Only one study used the Drug Abuse Screen Test (DAST-10) questionnaire to measure drug use (Talih et al., 2018).

As for measuring academic burnout, the Maslach Burnout Inventory (MBI) was the most used instrument in six studies (Cecil et al., 2014; Jackson et al., 2016; Lee et al., 2020; Mache et al., 2015; Vilchez-Cornejo et al., 2016; Zivojinović et al., 2020), and its version for students, Maslach Burnout Inventory - student version (MBI-SS), in another six studies (Arango Agudelo et al., 2010; Calcides 
et al., 2019; M.S. Carlotto et al., 2010; Erschens et al., 2018; Nteveros et al., 2020; Tavares et al., 2020). The Oldenburg Burnout Inventory (OLBI) academic version was used in three studies (Mbanga et al., 2018; Njim et al., 2018, 2019). Only one study used the Burnout Measure short version (BMS) instrument (Talih et al., 2018).

Table 2 shows the prevalence of consumption of alcohol, illicit substances and burnout syndrome in the studies analyzed, as well as the relationship that is object of this review. In studies that assessed the consumption or not of alcohol by students, there was a variation in consumption of $15.1 \%$ (Erschens et al., 2018) and $68.2 \%$
(Arango Agudelo et al., 2010). Abusive consumption/ dependence ranged from $9.2 \%$ (Talih et al., 2018) to $41.4 \%$ (Arango Agudelo et al., 2010). One study did not present the alcohol consumption frequency (Zivojinović et al., 2020).

With regard to illicit substances, of the eight studies that carried out their assessment, the report of use, regardless of the type of substance, ranged between $1.13 \%$ (Njim et al., 2018) and 34.9\% (Talih et al., 2018). Cannabis (marijuana) was the most reported drug by studies that specifically assessed the substances used (Arango Agudelo et al., 2010; Mache et al., 2015; Talih et al., 2018).

Table 2

Summary of the prevalence of consumption of alcohol, illicit substances and burnout syndrome and main results of associations between variables

\begin{tabular}{|c|c|c|c|c|}
\hline $\begin{array}{l}\text { Author } \\
\text { (year) }\end{array}$ & Alcohol consumption & $\begin{array}{l}\text { Consumption of } \\
\text { illicit substances }\end{array}$ & Prevalence of burnout syndrome & $\begin{array}{l}\text { Consumption of alcohol } \\
\text { or illicit substances x } \\
\text { burnout syndrome }\end{array}$ \\
\hline $\begin{array}{c}\text { Arango } \\
\text { Agudelo et al. } \\
(2010)\end{array}$ & $\begin{array}{l}68.2 \% \text { yes; } C A G E: 58.6 \% \text { non- } \\
\text { dependent, } 25.3 \% \text { dependent; } \\
16.1 \% \text { probable dependence. }\end{array}$ & $\begin{array}{l}10.5 \% \text { yes; } \\
45.0 \% \text { cannabis; } \\
23.5 \% \text { others }\end{array}$ & $\begin{array}{c}E E: 19.5 \% \text { (low); } 38.2 \% \text { (moderate); } 42.3 \% \\
\text { (high); } D P: 35.7 \% \text { (low); } 35.7 \% \text { (moderate); } \\
28.6 \% \text { (high); } P A: 39.0 \% \text { (high); } 33.0 \% \\
\text { (moderate); } 28.0 \% \text { (low) }\end{array}$ & $\begin{array}{l}\text { Use of psychoactive } \\
\text { substances associated with } \\
\text { high levels of DP }\end{array}$ \\
\hline $\begin{array}{l}\text { Calcides et al. } \\
\text { (2019) }\end{array}$ & - & $21.7 \%$ yes & $\begin{array}{l}10.3 \% \text { two-dimensional (EE; DP); } 35.9 \% \\
\text { three-dimensional; } E E: 17.9 \% \text { (light); } 23.4 \% \\
\text { (moderate); } 53.3 \% \text { (high); DP: } 13.6 \% \text { (light); } \\
31.5 \% \text { (moderate); } 52.2 \% \text { (high); PA: } 53.3 \% \\
\text { (high); } 22.8 \% \text { (moderate); } 19.0 \% \text { (light) }\end{array}$ & $\begin{array}{c}\text { Use of psychoactive } \\
\text { substances associated with BS }\end{array}$ \\
\hline $\begin{array}{l}\text { M.S. Carlotto } \\
\text { et al.(2010) }\end{array}$ & Average $1.27 \pm 0.57$ & - & $\begin{array}{l}\text { EE: mean } 2.50(\mathrm{sd} 1.45) ; D P: \text { mean } 1.20 \\
\quad(\mathrm{sd} 1.12) ; P R: \text { mean } 5.14(\mathrm{sd} 0.78)\end{array}$ & $\begin{array}{l}\text { Alcohol use was associated } \\
\text { with the highest average } \\
\text { of EE }\end{array}$ \\
\hline $\begin{array}{l}\text { Cecil et al. } \\
\text { (2014) }\end{array}$ & $\begin{array}{l}\text { 1st year: } 38.47 \pm 13.92 ; 2 \text { nd year: } \\
32.44 \pm 8.68 ; 3 \text { rd year: } 37.45 \pm \\
7.75 ; \text { 4th year: } 32.65 \pm 8.68\end{array}$ & - & $\begin{array}{c}26.7 \% \text { total; } E E: 54.8 \% \text { (high); } D P: 34.0 \% \\
\text { (high); } P A: 46.6 \% \text { (low) }\end{array}$ & $\begin{array}{l}\text { Alcohol use associated with } \\
\text { higher PA scores }\end{array}$ \\
\hline $\begin{array}{l}\text { Erschens et al. } \\
\qquad(2018)\end{array}$ & $15.1 \%$ yes & $\begin{array}{l}\text { Stimulant drugs: } \\
4.5 \% \text {; Tranquilizer } \\
\text { drugs: } 6.7 \%\end{array}$ & $\begin{array}{c}32.6 \% \text { total; } E E: 23.5 \% \text { (high); } D D: 17.8 \% \\
\text { (high); } P A: 35.8 \% \text { (low) }\end{array}$ & $\begin{array}{l}\text { BS associated with the use of } \\
\text { alcohol and stimulant drugs }\end{array}$ \\
\hline $\begin{array}{l}\text { Jackson et al. } \\
(2016)\end{array}$ & $32.4 \%$ alcohol abuse/dependence & - & Does not present descriptive data & $\begin{array}{l}\text { Alcohol use associated with } \\
\text { EE and DP }\end{array}$ \\
\hline $\begin{array}{l}\text { Lee et al. } \\
\text { (2020) }\end{array}$ & $\begin{array}{c}\text { Frequency: Never: } 29.7 \% ; 2-4 \mathrm{x} / \\
\text { month: } 18.3 \% ; 2-3 \mathrm{x} / \text { week: } 5.2 \% \text {; } \\
\text { 4-5x/week: } 1.4 \% \\
\text { Abusive consumption: Never: } \\
73.1 \% ; 26.9 \% \text { yes }\end{array}$ & - & $\begin{array}{c}27.9 \% \text { total; } E E: 21.6 \% \text { (low), } 29.2 \% \\
\text { (moderate), } 49.3 \% \text { (high); DP: } 23.4 \% \text { (low), } \\
22.8 \% \text { (moderate), } 53.8 \% \text { (high); } P A: 5.1 \% \\
\text { (high), } 23.2 \% \text { (moderate) }\end{array}$ & $\begin{array}{c}\text { BS associated with greater } \\
\text { chance of using alcohol 4-5 } \\
\text { x a week }\end{array}$ \\
\hline $\begin{array}{l}\text { Mache et al. } \\
\quad(2015)\end{array}$ & $53.0 \%$ yes & $\begin{array}{l}\text { Cannabis: } 8.0 \% \text {; } \\
\text { Cocaine: } 0.8 \% \text {; } \\
\text { Ecstasy: } 0.4 \%\end{array}$ & $\begin{array}{c}E E: \text { mean } 18.1 \pm 8.8 ; D P: \text { mean } 6.5 \pm 4.8 ; P A: \\
\text { mean } 31.3 \pm 7.9\end{array}$ & $\begin{array}{l}\text { Cannabis use associated } \\
\text { with high DP, stimulant use } \\
\text { associated with low PA }\end{array}$ \\
\hline $\begin{array}{l}\text { Mbanga et al. } \\
\text { (2018) }\end{array}$ & - & $1.64 \%$ yes & $37.58 \%$ total & $\begin{array}{l}\text { Use of recreational drugs } \\
\text { associated with BS }\end{array}$ \\
\hline $\begin{array}{l}\text { Njim et al. } \\
(2019)\end{array}$ & $30.0 \%$ yes & $2.21 \%$ yes & $E E$ : mean $16.64 \pm 3.39 ; D P$ : mean $20.49 \pm 3.53$ & $\begin{array}{l}\text { Use of alcohol and recreational } \\
\text { drugs associated with BS }\end{array}$ \\
\hline $\begin{array}{l}\text { Njim et al. } \\
\text { (2018) }\end{array}$ & $28.48 \%$ yes & $1.13 \%$ yes & $E E:$ mean $20.94 \pm 3.04 ; D P:$ mean $17.10 \pm 3.09$ & $\begin{array}{c}\text { There was no association } \\
\text { between } B S \text {, alcohol and } \\
\text { drug use }\end{array}$ \\
\hline $\begin{array}{l}\text { Nteveros et al. } \\
\text { (2020) } \\
\text { Talih et al. } \\
\quad(2018)\end{array}$ & $\begin{array}{c}44.2 \% \text { yes } \\
58.1 \% \text { yes } \\
9.2 \% \text { abusive consumption }\end{array}$ & $\begin{array}{c}- \\
34.9 \% \text { yes; } 31.9 \% \\
\text { cannabis. DAST: } \\
1.7 \% \text { severe } \\
\text { problem; } 8.3 \% \\
\text { moderate problem; } \\
\text { 43.4\% light problem }\end{array}$ & $\begin{array}{c}18.1 \% \text { total; } 51.1 \% 1 \text { dimension; } 23.6 \% 2 \\
\text { dimensions; } 7.1 \% \text { in all dimensions } \\
43.0 \% \text { total }\end{array}$ & $\begin{array}{c}\text { There was no association } \\
\text { between BS and alcohol use. } \\
\text { There was no association } \\
\text { between BS, alcohol and } \\
\text { substance use }\end{array}$ \\
\hline $\begin{array}{l}\text { Tavares et al. } \\
\quad(2020)\end{array}$ & $62.5 \%$ yes & $\begin{array}{l}25.1 \% \text { sometimes } \\
9.3 \% \text { always }\end{array}$ & $\begin{array}{c}\text { EE: } 3.6 \pm 1.2 \text {; High EE: } 38.4 \% \\
\text { DP: } 1.7 \pm 1.4 \text {; High DP: } 29.4 \% \\
\text { PE: } 4.3 \pm 0.9 \text {; Low PE: } 32.7 \% \\
\text { BS: } 9.5 \%\end{array}$ & $\begin{array}{l}\text { Alcohol use associated with } \\
\mathrm{EE} \text { and PE and stimulant use } \\
\text { associated with EE }\end{array}$ \\
\hline $\begin{array}{l}\text { Vilchez- } \\
\text { Cornejo et al. } \\
\quad(2016)\end{array}$ & $36.9 \%$ overall & - & $\begin{array}{c}24.1 \% \text { with two or more components; } E E: \\
52.5 \% \text { (light); } 31 \% \text { (moderate); } 16.5 \% \text { (high); } \\
D P: 30.5 \% \text { (light); } 21.8 \% \text { (moderate), } 47.7 \% \\
\text { (high); PA: } 42.7 \% \text { (high); } 22.0 \% \text { (average); } \\
35.3 \% \text { (low). }\end{array}$ & $\begin{array}{l}\text { Alcohol use associated with } \\
\text { two or more BS components }\end{array}$ \\
\hline $\begin{array}{l}\text { Zivojinovic } \\
\text { et al. (2020) }\end{array}$ & Does not present descriptive data & - & $\begin{array}{c}43.3 \% \text { total; } \\
\text { EE: mean } 25.60 \pm 11.61(45.0 \% \text { high }) ; \\
\text { DP: mean } 15.40 \pm 6.56(79.4 \% \text { high }) ; \\
\boldsymbol{P A}: \text { mean } 32.18 \pm 8.50(50.5 \% \text { low }) \\
\end{array}$ & $\begin{array}{l}\text { There was no association } \\
\text { between BS and alcohol use. }\end{array}$ \\
\hline
\end{tabular}

Note. $\mathrm{DP}=$ depersonalization; $\mathrm{EE}=$ emotional exhaustion; $\mathrm{PE}=$ Professional Effectiveness; $\mathrm{PA}=$ personal achievement; $\mathrm{BS}=$ burnout syndrome. DAST $=$ The Drug Abuse Screening Test. 
To analyze the burnout syndrome, some studies used relative frequencies (Arango Agudelo et al., 2010; Cecil et al., 2014; Erschens et al., 2018; Lee et al., 2020; Mbanga et al., 2018; Nteveros et al., 2020; Talih et al., 2018; Tavares et al., 2020; Vilchez-Cornejo et al., 2016), others used means with standard deviation (M.S. Carlotto et al., 2010; Mache et al., 2015; Njim et al., 2018, 2019; Tavares et al., 2020; Zivojinović et al., 2020). Only one study did not present the prevalence of $\mathrm{BS}$, and according to the author, these rates have already been presented in another study (Jackson et al., 2016).

For studies that evaluated burnout syndrome as a whole (Calcides et al., 2019; Cecil et al., 2014; Erschens et al., 2018; Lee et al., 2020; Mbanga et al., 2018; Nteveros et al., 2020; Talih etal., 2018; Vilchez-Cornejo etal., 2016;Zivojinović etal., 2020), a variation of $9.5 \%$ (Tavares et al., 2020) to $43.3 \%$ (Zivojinović et al., 2020) was perceived. Regarding associations, the main objective of this review, seven studies verified the relation of the BS with the use of alcoholic beverages, two studies with the use of illicit substances, and seven studies with both outcomes. Of the sixteen studies included in this review, twelve found an association between the variables.

Alcohol consumption was shown to be strongly related to BS, and some found an association with BS in general, without specifying the domains (Erschens et al., 2018; Lee et al., 2020; Njim et al., 2019; Vilchez-Cornejo et al., 2016) and others found an association with different dimensions of burnout, as in the study by Jackson et al. (2016), who identified an association between EE and DP with alcohol consumption, by Tavares et al. (2020), who identified an association between $\mathrm{EE}$ and Professional Effectiveness (PE) with alcohol consumption and Cecil et al. (2014), who identified an opposite relation to the others, showing that alcohol consumption generated a higher PA score, decreasing the chances of burnout.

As for the use of illicit substances, this was associated with BS in general, without specifying domains, in four studies (Calcides et al., 2019; Erschens et al., 2018; Mbanga et al., 2018; Njim et al., 2018; Njim et al., 2019). Three other studies found an association with different dimensions of burnout, such as Arango Agudelo et al. (2010), who demonstrated that students who consume substances showed higher levels of DP, Mache et al. (2015), who found higher levels of DP in students who used cannabis and lower levels of PA in students who used stimulant drugs and in the study by Tavares et al. (2020), who found an association between EE and the use of stimulants. Four studies found no association between alcohol consumption and BS (Njim et al., 2018; Nteveros et al., 2020; Zivojinović et al., 2020), and two of these also assessed the use of illicit substances and found no significant association (Njim et al., 2018; Talih et al., 2018).

\section{Discussion}

The main objective of this study was to synthesize the scientific evidence related to the consumption of alcohol and illicit substances associated with burnout syndrome in university students. All studies found evaluated students in the health field, with emphasis on the Medicine course. There is greater concern with students in the health professions due to the characteristics of these courses, which require more time, great early responsibility, in addition to the high physical and psychological involvement with people's illness and suffering, which makes these students more vulnerable in the development of stress and emotional exhaustion (Murakami, Panúncio-Pinto, Santos, \& Troncon, 2019; Padovani et al., 2014).

As there was an option for undergraduate courses in the health area, which are more subject to emotional exhaustion, the sample size was limited and without great variation, with the majority having populations below 900 individuals. Samples with smaller numbers of participants have reduced statistical analysis power, and may generate different results in the associations between variables (Luiz \& Magnanini, 2000). The great diversity of countries where the studies were conducted can interfere in the way undergraduate students behave in relation to the consumption of alcohol and illicit substances, due to the different social, cultural, economic and religious aspects of each region, which may bring greater or less stigma on the use of these substances (Khalili et al., 2018; Sanchez \& Nappo, 2007) and, consequently, affect the relationship with the burnout syndrome, which can be verified in studies carried out in Cyprus (Nteveros et al., 2020), Lebanon (Talih et al., 2018) and Serbia (Zivojinović et al., 2020).

The way to measure the consumption of alcoholic beverages and illicit substances was different. The AUDIT or AUDIT-C questionnaire are specific WHO instruments to assess alcohol consumption in the last 12 months (WHO, 2001). The AUQ assesses the desire and urgency to consume alcoholic beverages (Bohn, Krahn, \& Staehler, 1995). The CAGE questionnaire measures alcohol consumption through four questions about the need to quit (C-cutting-down), criticism of consumption (A-annoyance), guilt (G-guilty) and the need for consumption at the beginning of the morning (Eeye-openers) (Mayfield, McLeod, \& Hall, 1974). And the COPE inventory is used to verify coping strategies, divided into 15 scales, one of which is the use of substances to mitigate stressful effects, however, this includes the use of alcohol and medication and does not make it clear which substance was used (Carver, Scheier, \& Weintraub, 1989). Although the literature presents several instruments to measure the use of alcohol, most of the studies used the simple questioning methodology, which was also verified with regard to the consumption of illicit substances. The DAST-10 was the only specific instrument to assess drug use, and this instrument assesses the use of various substances (including tranquilizers) in the last 12 months (Yudko, Lozhkina, \& Fouts, 2007).

The prevalence of alcohol and illicit substance consumption varied widely among the studies analyzed, and this difference may be related to the different instruments for measuring the variables or to the methodology used for data collection. In addition, some authors pointed out the 
issue of the sample residing in very conservative countries, where the use of these substances is stigmatized ( Njim et al., 2018; Talih et al., 2018). It was also found that some instruments that measure the use of illicit substances included medications in their assessment, which can confound the findings or increase the prevalence (Arango Agudelo et al., 2010; Mbanga et al., 2018; Njim et al., 2018).

As for burnout syndrome, the MBI and MBI-SS, the main instruments used, are divided into three subscales; EE, DP directly related to burnout, so the higher the score, the greater the burnout - and PA - that the lower the score, the greater the burnout experience (Maslach \& Jackson, 1981). The Oldenburg Burnout Inventory (OLBI) assesses two components: exhaustion (physical, affective and cognitive) and disengagement, excluding personal achievement (Reis, Xanthopoulou, \& Tsaousis, 2015). Burnout syndrome also showed great variation among the populations evaluated, and the difference in the form of measurement, in addition to the use of instruments not adapted for the academic population, may contribute to different findings.

It is noteworthy, as already mentioned, that 12 studies identified the association between burnout syndrome and the consumption of alcohol and/or illicit substances, which suggests that situations of emotional exhaustion can lead university students to resort to the use of psychoactive drugs as a way of coping with adversity. The use of these substances is a method of confrontation with an emotional focus, as the student seeks to interfere with the personal ability to assess stressful situations through an escape mechanism. The use of this method generates an immediate but short-term relief response (R.C. Carlotto, Teixeira, \& Dias, 2015; Pietrowski, Cardoso, \& Bernardi, 2018).

The study by Cecil et al. (2014) was the only one to find an opposite relationship between burnout syndrome and alcohol consumption. The authors mention that there is evidence that alcohol consumption can be used as a strategy for coping with stress and emotional exhaustion, however, it is suggested that alcohol use in the population may be related to the pleasure of drinking and socializing, and not as a coping mechanism. It is important to note that this was the only study that adopted the AUQ questionnaire to measure alcohol consumption in students, in addition to not using the MBI adapted for students, which suggests the need for further studies to verify whether these instruments are suitable to be used in this population.

Regarding studies that did not find a significant association between burnout syndrome and the consumption of alcohol and/or illicit substances, Njim et al. (2018) emphasizes that as their study was conducted in a society in which the use of these substances is extremely disapproved, students may have omitted their use in an attempt to be socially accepted. Talih et al. (2018) attributes the non-association of BS with the use of alcohol and illicit drugs to the higher level of awareness about the consumption of these substances by medical students, the stigma of use in the Lebanese culture, the sample size and the fear of exposing the consumption. Nteveros et al. (2020) and Zivojinović (2020) reported that, despite the lack of association between BS and alcoholic beverages, students who consumed alcohol had higher scores in burnout components, indicating greater consumption of these substances in students with higher levels of exhaustion, hypothesis suggested by this investigation.

The burnout syndrome is still unknown among some professionals and undergraduate students, and there is a lack in the search for programs aimed at detecting, preventing or helping students who go through this situation, making actions in higher education institutions aimed at health education and assistance to university students in order to avoid the consequences that academic exhaustion presents (Lopes \& Guimarães, 2016; Rees et al., 2016; Silveira et al., 2016).

In conclusion, burnout syndrome is associated with the use of alcohol and illicit substances, as shown in twelve of the sixteen studies found, and it may be a negative strategy for coping with academic exhaustion, although the small number of studies and the use of instruments not validated for the studied population may compromise the completeness of these conclusions. Also, due to the cross-sectional design of the studies found, temporal associations cannot be verified, leading to the risk of reverse causality. In this sense, more research is needed, with robust methodologies, especially with a longitudinal design, to verify whether the use of alcohol and drugs directly results in academic burnout or students with high levels of exhaustion resort to the use of these substances as a coping strategy. It is important that studies be conducted with other undergraduate courses in addition to the health area, in order to identify whether students from other educational areas are also vulnerable to the stress provided by the academic environment and its implications for behaviors that are harmful to health.

\section{References}

Arango Agudelo, S., Castaño Castrillón, J. J., Henao Restrepo, C. J., Jimenez Aguirre, D. P., López Henao, A. F., \& Páez Cala, M. L. (2010). Síndrome de Burnout y factores asociados en estudiantes de I a X semestre de la Facultad de Medicina de la Universidad de Manizales (Colombia), 2009 [Burnout syndrome and associated factors, in students from I to X semester of the Faculty of Medicine at the University of Manizales (Colombia), 2009]. Archivos de Medicina, 10(2), 110-126. doi:10.30554/archmed.10.2.1291.2010

Aresi, G., Moore, S., \& Marta, E. (2016). Drinking, drug use, and related consequences among university students completing study abroad experiences: A systematic review. Substance Use \& Misuse, 51(14), 1888-1904. doi:10.1080/10826084.2016.1201116

Ariño, D. O., \& Bardagi, M. P. (2018). Relação entre fatores acadêmicos e a saúde mental de estudantes universitários [Relationship between academic factors and mental health of university students]. Psicologia em Pesquisa, 12(3), 44-52. doi:10.24879/2018001200300544 
Benincasa, M., Tavares, A. L., Barbosa, V. M. M., Lajara, M. P., Rezende, M. M., Heleno, M. G. V., \& Custódio, E. M. (2018). A influência das relações e o uso de álcool por adolescentes [The influence of relationships and the use of alcohol by adolescents]. SMAD Revista Eletrônica Saúde Mental Álcool e Drogas, 14(1), 5-11. doi:10.11606/issn.1806-6976.smad.2018.000357

Bohn, M. J., Krahn, D. D., \& Staehler, B. A. (1995). Development and initial validation of a measure of drinking urges in abstinent alcoholics. Alcoholism: Clinical and Experimental Research, 19(3), 600-606. doi:10.1111/j.1530-0277.1995.tb01554.x

Calcides, D. A. P., Didou, R. N., Melo, E. V., \& Oliva-Costa, E. F. (2019). Burnout Syndrome in medical internship students and its prevention with Balint Group. Revista da Associação Médica Brasileira, 65(11), 1362-1367. doi:10.1590/1806-9282.65.11.1362

Carlotto, M. S., Câmara, S. G., Otto, F., \& Kauffmann, P. (2010). Estudo da Síndrome de Burnout em estudantes de psicologia [Study of Burnout Syndrome in psychology students]. Boletim de Psicologia, 59(131), 167-178. Retrieved from http://pepsic.bvsalud.org/pdf/bolpsi/ v59n131/v59n131a04.pdf

Carlotto, R. C., Teixeira, M. A. P., \& Dias, A. C. G. (2015). Adaptação acadêmica e coping em estudantes universitários [Academic adaptation and coping in university students]. Psico-USF, 20(3), 421-432. doi:10.1590/1413-82712015200305

Carver, C. S., Scheier, M. F., \& Weintraub, K. J. (1989). Assessing coping strategies: A theoretically based approach. Journal of Personality and Social Psychology, 56(2), 267-283. doi:10.1037/0022-3514.56.2.267

Cecil, J., McHale, C., Hart, J., \& Laidlaw, A. (2014). Behaviour and burnout in medical students. Medical Education Online, 19, 25209. doi:10.3402/meo.v19.25209

Davoren, M. P., Demant, J., Shiely, F., \& Perry, I. J. (2016). Alcohol consumption among university students in Ireland and the United Kingdom from 2002 to 2014: A systematic review. BMC Public Health, 16, 173. doi:10.1186/s12889-016-2843-1

Erschens, R., Loda, T., Herrmann-Werner, A., Keifenheim, K. E., Stuber, F., Nikendei, C., ... Junne, F. (2018). Behaviour-based functional and dysfunctional strategies of medical students to cope with burnout. Medical Education Online, 23(1), 1535738. doi:10.1080/10872981.2018.1535738

Jackson, E. R., Shanafelt, T. D., Hasan, O., Satele, D. V., \& Dyrbye, L. N. (2016). Burnout and alcohol abuse/dependence among U.S. medical students. Academic Medicine, 91(9), 1251-1256. doi:10.1097/ACM.0000000000001138
Khalili, M., Rahimi-Movaghar, A., Shadloo, B., Mojtabai, R., Mann, K., \& Amin-Esmaeili, M. (2018). Global scientific production on illicit drug addiction: A twodecade analysis. European Addiction Research, 24(2), 60-70. doi:10.1159/000487590

Lee, K. P., Yeung, N., Wong, C., Yip, B., Luk, L., \& Wong, S. (2020). Prevalence of medical students' burnout and its associated demographics and lifestyle factors in Hong Kong. PLoS One, 15(7), e0235154. doi:10.1371/journal.pone. 0235154

Lopes, F. L., \& Guimarães, G. S. (2016). Estudo da Síndrome de Burnout em estudantes de psicologia [Study of Burnout Syndrome in psychology students]. Psicologia: Ensino \& Formação, 7(1), 40-58. doi:10.21826/217958002016714058

Luiz, R. R., \& Magnanini, M. M. F. (2000). A lógica da determinação do tamanho da amostra em investigações epidemiológicas [The logic of determining sample size in epidemiological investigations]. Cadernos de Saúde Coletiva, 8(2), 9-28. Retrieved from http://www. cadernos.iesc.ufrj.br/cadernos/images/csc/2000_2/ artigos/csc_v8n2_09-28.pdf

Mache, S., Vitzthum, K., \& Groneberg, D. A. (2015). Prevention of study-related stress symptoms: Healthpromoting behavior among dental students. Wiener Medizinische Wochenschrift, 165(5-6), 100-106. doi:10.1007/s10354-014-0341-6

Maslach, C., \& Jackson, S. E. (1981). The measurement of experienced burnout. Journal of Organizational Behavior, 2(2), 99-113. doi:10.1002/job.4030020205

Maslach, C., Jackson, S. E., \& Leiter, M. P. (1997). The Maslach Burnout Inventory Manual. In C. P. Zalaquett \& R. J. Wood (Eds.), Evaluating stress: A book of resources (pp. 191-218). Lanham, MD: The Scarecrow Press.

Mayfield, D., McLeod, G., \& Hall, P. (1974). The CAGE Questionnaire : Validation of a new alcoholism screening instrument. American Journal of Psychiatry, 131(10), 1121-1123. doi:10.1176/ajp.131.10.1121

Mbanga, C. M., Efie, D. T., Aroke, D., \& Njim, T. (2018). Prevalence and predictors of recreational drug use among medical and nursing students in Cameroon: A cross sectional analysis. BMC Research Notes, 11(1), 515. doi:10.1186/s13104-018-3631-z

Munhoz, T. N., Santos, I. S., Nunes, B. P., Mola, C. L., Silva, I. C. M., \& Matijasevich, A. (2017). Tendências de consumo abusivo de álcool nas capitais brasileiras entre os anos de 2006 a 2013: Análise das informações do VIGITEL [Trends in alcohol abuse in Brazilian capitals between 2006 and 2013: Analysis of VIGITEL information]. Cadernos de Saúde Pública, 33(7), e00104516. doi:10.1590/0102-311x00104516 
Murakami, K., Panúncio-Pinto, M. P., Santos, J. L. F., \& Troncon, L. E. A. (2019). Estresse psicológico em estudantes de cursos de graduação da área da saúde [Psychological stress in undergraduate health students courses] Revista de Medicina (São Paulo), 98(2), 108-113. doi:10.11606/issn.1679-9836.v98i2p108-113

Newman, I., Ding, L., \& Feng, Y. (2017). Estimate of undergraduate university student alcohol use in China: A systematic review and meta-analysis. Archives of Public Health, 75, 52. doi:10.1186/s13690-017-0220-x

Njim, T., Mbanga, C., Mouemba, D., Makebe, H., Toukam, L., Kika, B., \& Mulango, I. (2018). Determinants of burnout syndrome among nursing students in Cameroon: Cross-sectional study. BMC Research Notes, 11(1), 450. doi:10.1186/s13104-018-3567-3

Njim, T., Makebe, H., Toukam, L., Kika, B., Fonkou, S., Fondungallah, J., \& Fondong, A. (2019). Burnout Syndrome amongst medical students in Cameroon: A cross-sectional analysis of the determinants in preclinical and clinical students. Psychiatry Journal, 2019, 4157574. doi:10.1155/2019/4157574

Nteveros, A., Kyprianou, M., Artemiadis, A., Charalampous, A., Christoforaki, K., Cheilidis, S., ... Zis, P. (2020). Burnout among medical students in Cyprus: A cross-sectional study. PLoS One, 15(11), e0241335. doi:10.1371/journal.pone.0241335

Pacheco, J. P., Giacomin, H. T., Tam, W. W., Ribeiro, T. B., Bezerra, C. A. M., \& Pinasco, G. C. (2017). Mental health problems among medical students in Brazil: A systematic review and meta-analysis. Revista Brasileira de Psiquiatria, 39(4), 369-378. doi:10.1590/1516-4446-2017-2223

Padovani, R. C., Neufeld, C. B., Maltoni, J., Barbosa, L. N. F., Souza, W. F., Cavalcanti, H. A. F., \& Lameu, J. N. (2014). Vulnerabilidade e bem-estar psicológicos do estudante universitário [Vulnerability and psychological well-being of university student]. Revista Brasileira de Terapias Cognitivas, 10(1), 2-10. doi:10.5935/1808-5687.20140002

Pietrowski, D. L., Cardoso, N. O., \& Bernardi, C. C. N. (2018). Estratégias de coping frente à síndrome de burnout entre os professores: Uma revisão integrativa da literatura nacional [Coping strategies against the burnout syndrome among teachers: An integrative review of the national literature]. Contextos Clínicos, 11(3), 397-409. doi:10.4013/ctc. 2018.113 .10

Rees, C. S., Heritage, B., Osseiran-Moisson, R., Chamberlain, D., Cusack, L., Anderson, J., ... Hegney, D. G. (2016). Can we predict burnout among student nurses? An exploration of the ICWR-1 model of individual psychological resilience. Frontiers in Psychology, 7, 1072. doi:10.3389/fpsyg.2016.01072
Reis, D., Xanthopoulou, D., \& Tsaousis, I. (2015). Measuring job and academic burnout with the Oldenburg Burnout Inventory (OLBI): Factorial invariance across samples and countries. Burnout Research, 2, 8-18. doi:10.1016/j.burn.2014.11.001

Sanchez, Z. M., \& Nappo, S. A. (2007). Religiosity, spirituality and psychotropic drug use. Revista de Psiquiatria Clínica, 34(supl. 1), 73-81. doi:10.1590/S0101-60832007000700010

Silveira, A. L. P., Della Colleta, T. C., Ono, H. R. B., Woitas, L. R., Soares, S. H., Andrade, V. L. A., \& Araújo, L. A. (2016). Síndrome de Burnout: Consequências e implicações de uma realidade cada vez mais prevalente na vida dos profissionais de saúde [Burnout Syndrome: Consequences and implications of an increasingly prevalentreality in the lives of health professionals] Revista Brasileira de Medicina do Trabalho, 14(3), 275-284. doi:10.5327/Z1679-443520163215

Talih, F., Daher, M., Daou, D., \& Ajaltouni, J. (2018). Examining Burnout, depression, and attitudes regarding drug use among Lebanese medical students during the 4 years of medical school. Academic Psychiatry, 42(2), 288-296. doi:10.1007/s40596-017-0879-x

Tavares, H. H. F., Silva, H. R. S., Miranda, I. M. M., Braga, M. S., Santos, R. O., \& Guerra, H. S. (2020). Factors associated with Burnout Syndrome in medical students. Mundo da Saúde, 44(1), 280-289. doi:10.15343/0104-7809.202044280289

Trevisan, E. R., \& Castro, S. S. (2019). Centros de Atenção Psicossocial - álcool e drogas: Perfil dos usuários [Psychosocial Care Centers - Alcohol and drugs: Profile of users]. Saúde em Debate, 43(121), 450-463. doi:10.1590/0103-1104201912113

United Nations Office on Drugs and Crime. (2020). World Drug Report: Drug use and health consequences. Vienna, Austria: United Nations. Retrieved from https://wdr.unodc. org/wdr2020/field/WDR20_Booklet_2.pdf

Vilchez-Cornejo, J., Huamán-Gutiérrez, R., ArceVillalobos, L., Morán-Mariños, C., MihashiroMaguiña, K., Melo-Mallma, N., ... Rojas-Valle, C. (2016). Síndrome de burnout en estudiantes de medicina: Frecuencia, características y factores asociados [Burnout syndrome in medical students: Frequency, characteristics and associated factors] Acta Médica Peruana, 33(4), 282-288. doi:10.35663/amp.2016.334.221

World Health Organization. (2001). The Alcohol Use Disorders Identification Test (AUDIT). Geneva, Switzerland: WHO.

World Health Organization. (2018). Global status report on alcohol and health 2018. Geneva, Switzerland: WHO. Retrieved from https://apps.who.int/iris/bitstream/hand le/10665/274603/9789241565639-eng.pdf 
Yudko, E., Lozhkina, O., \& Fouts, A. (2007). A comprehensive review of the psychometric properties of the Drug Abuse Screening Test. Journal of Substance Abuse Treatment, 32(2), 189-198. doi:10.1016/j.jsat.2006.08.002

Zivojinović, J. I., Backović, D., Belojević, G., Valčić, O., Soldatović, I., \& Janković, J. (2020). Predictors of burnout among Belgrade veterinary students: A cross-sectional study. PLoS One, 15(3), e0230685. doi:10.1371/journal.pone.0230685

Giovana Frazon Andrade is a Professor of the Universidade Estadual do Centro-Oeste, Guarapuava-PR, Brazil.

Poliana Vieira da Silva Menolli is a Professor of the Universidade Estadual do Oeste do Paraná, Cascavel-PR, Brazil.

Pedro Augusto Clemente is a graduation student of the Universidade Estadual do Centro-Oeste, Guarapuava-PR, Brazil.

Arthur Eumann Mesas is a Professor of the Universidad de Castilla-La Mancha, Cuenca, Espanha.

Dannyele Cristina Silva is a Professor of the Universidade Estadual do Centro-Oeste, Guarapuava-PR, Brazil.

Edmarlon Girotto is a Professor of the Universidade Estadual de Londrina, Londrina-PR, Brazil.

Authors' Contribution:

All authors made substantial contributions to the conception and design of this study, to data analysis and interpretation, and to the manuscript revision and approval of the final version. All the authors assume public responsability for content of the manuscript.

Associate Editor:

Wanderlei Abadio de Oliveira

Received: Apr. 06, 2021

1st Revision: May. 17, 2021

Approved: Jun. 24, 2021

How to cite this article:

Andrade, G. F., Menolli, P. V. S., Clemente, P. A., Mesas, A. E., Silva, D. C., \& Girotto, E. (2021). Burnout syndrome and consumption of alcohol and illicit substances in university students. Paidéia (Ribeirão Preto), 31, e3134. doi:https://doi.org/10.1590/1982-4327e3134 